\title{
A possible effect of B-chromosomes on metaphase I homologous chromosome association in rye
}

\author{
M. T. AlVAREZ, A. FOMINAYA, * M. PEREZ DE LA VEGA, \\ Departamento de Genética, Facultad de Biologia, Universidad de León, E-24071 Léon, *Departamento de Genética, \\ Facultad de Ciencias, Universidad de Alcalá de Henares, E-28871 A/calá de Henares, Spain
}

\begin{abstract}
The metaphase I association of rye chromosomes was examined both in normal plants $(2 n=14)$ and in plants with rye $\mathrm{B}$ chromosomes $(2 n=14+\mathrm{Bs})$ using the $\mathrm{C}$-banding technique. It was found that $\mathrm{B}$ chromosomes (Bs) affect homologous chromosome association. $\mathrm{B}$ isochromosomes (iso- $\mathrm{Bs}$ ) were present in several plants; their presence was deduced, in addition to the morphology of chromosomes at mitosis, from the meiotic association between the two arms of the B and it was confirmed by its $\mathrm{C}$-banding pattern. The strongest increase in rye metaphase association (measured as bound arms) was produced by the presence of the iso-B short (iso-BS) chromosome. The comparison of chromosome association between plants with and without Bs seems to point to the existence of a promoting effect on meiotic chromosome association by Bs, and in particular by the short arm.
\end{abstract}

Keywords: B-chromosome, chromosome association, rye, Secale cereale.

\section{Introduction}

Although aneuploidy is not a frequent event in nature, some eukaryotic taxa of both plants and animals have extra chromosomes, known as B chromosomes (Bs). These chromosomes constitute a somewhat heterogenous class which are clearly distinguished from the standard chromosome complement, or A chromosomes (As), in both morphology and behaviour, and As and Bs lack of homology. Therefore, the DNA which Bs contribute to the nucleus does not represent a precise copy of part of the normal nuclear DNA fraction as is the case with aneuploids. Bs are evidently dispensable, and not essential for normal development and reproduction. For this reason it is not surprising that they were initially considered to be inert, a view reinforced by the claim that Bs are frequently, but by no means always, heterochromatic (Jones \& Rees, 1982).

The addition or loss of individual chromosomes usually leads to gene unbalance which may result, among other effects, in a reduction of chiasma frequency. Several works in this line have been devoted to the study of the influence of Bs on the chromosome pairing and the chiasma frequency of As. The presence of Bs increasing chiasma frequency and genetic recombination of the As has been described in several works (Ayonoadu \& Rees, 1968; Tsumoto \& Sasaki, 1972; Parker et al., 1990), while in others a negative correlation or no correlation at all have been described (Jones \& Rees, 1967; Zarchi et al., 1972).

Not only chiasma frequency but also chromosome pairing between different genomes can be influenced by the presence of $\mathrm{Bs}$; thus, the Bs of some grass species can mimic the role of the wheat $P h$ gene in the absence of chromosome 5B of wheat (Dover \& Riley, 1972; Romero \& Lacadena, 1980).

The aim of this work was to study the influence of Bs on the homologous association at metaphase I of the chromosome complement of rye, both As and Bs, in plants from a rye population in which it has recently been demonstrated that Bs have an influence on the rate of outcrossing (Cruz-Pardilla et al., 1989).

\section{Materials and methods}

The material used was a population of the Japanese cultivar JNK of diploid rye (Secale cereale L., $2 n=14+$ Bs). A large sample of seeds was kindly provided by $\mathrm{Dr}$ Kishikawa and the population has been maintained and replanted several times in Spain.

Chromosome numbers were determined in root tips by conventional Feulgen-staining techniques. Plants carrying B chromosomes were grown under green- 
house conditions. Anthers for meiotic analysis were collected and fixed in alcohol-acetic acid 3:1. As metaphase I rye bivalents are not easily identified by standard staining techniques, a Giemsa C-banding procedure (Giráldez \& Orellana, 1979) was used to identify bivalents and bivalent arms at metaphase I. This technique has been extensively used to identify unambiguously rye bivalent arms in meiosis of rye and triticale (Fominaya \& Orellana, 1988; Benavente \& Orellana, 1989).

We have determined the degree of chromosome association by the number of bound arms at metaphase I (metaphase I bounds). Plants with the same chromosome constitution always showed an homogeneous chromosome association level (non-significant chisquare test).

In order to establish a possible difference between plants with and without Bs in the overall degree of metaphase I association, as well as in the participation of each chromosome pair, $2 \times 2$ contingency chi-square $\left(\chi^{2}, 1\right.$ d.g. $)$ tests were employed to compare the observed number of bound versus unbound arms between plant classes. We have scored the degree of chromosome association as bound arms, but our data can be compared with other data recorded as chiasma frequency substituting a chiasma for bound arm. The numbers of chiasmata and bounds recorded were very close in our material, as reported in other rye materials (Giráldez \& Orellana, 1979; Orellana \& Giráldez, 1981)

\section{Results}

The presence of large blocks of telomeric constitutive heterochromatin in one or both chromosome arms allows for the identification of the A chromosomes, which constitute the normal complement of rye. In addition to morphology, Giemsa staining has also enabled us to characterize rye $\mathrm{B}$ chromosomes, either standard or isochromosomes. The standard Bs of rye have a heterochromatin block in their long arm and a lack of heterochromatin in their short arm (Fig. 1).

The frequencies of chromosomes arms bound per cell observed in the plants analysed are shown in Table 1. From the data it can be inferred that, in general, and at least in the rye cultivar studied here, the presence of Bs induces an increase in homologous association in rye chromosomes, estimated as the mean of bound arms. The effect of $\mathrm{Bs}$ on the bound chromosome complement was estimated by comparing plants with and without $\mathrm{Bs}$ and among plants with different numbers using $2 \times 2$ contingency $\chi^{2}$ test. Significant differences were not found either in the comparisons between $2 n=14$ plants and both $2 n=14+2 \mathrm{~B}+$ iso-
$\mathrm{BL}$ and $2 n=14+$ iso-BL (Table 1), or in that between $2 n=14+2 \mathrm{~B}+$ iso-BL and $2 n=14+$ iso-BL, or between $2 n=14+2 \mathrm{~B}$ and $2 n=14+$ iso-BS $\left(\chi^{2}=2.12\right.$, $0.20>P>0.10 ; \quad \chi^{2}=0.08, \quad 0.80>P>0.70$, respectively, data not shown in Table 1). On the other hand, significant differences were observed in all other cases: $2 n=14$ plants versus $2 n=14+2 \mathrm{~B}$ or $2 n=14+$ iso-BS (Table 1), $2 n=14+2 \mathrm{~B}+$ iso-BL plants versus $2 n=14+2 \mathrm{~B} \quad$ or $\quad 2 n=14+$ iso-BS $\quad\left(\chi^{2}=29.01\right.$, $\left.P<0.001 ; \quad \chi^{2}=28.77, \quad P<0.001\right), 2 n=14+$ iso-BL versus $2 n=14+2 \mathrm{~B}$ or $2 n=14+$ iso-BS $\left(\chi^{2}=24.61\right.$, $\left.P<0.001 ; \chi^{2}=21.76, P<0.001\right)$. The significant differences in chromosome association were due to short arms, the differences between long arms being nonsignificant (Table 1). $\chi^{2}$ values for the comparisons between plants with $2 n=14$ and plants with $2 n=14+$ iso-BS and $2 n=14+2 \mathrm{~B}+$ iso-BL were not scored because of empty classes in the contingency tables.

The pertinent contingency tests between plants with and without Bs were carried out with the aim of discovering whether the significant differences observed in the chromosome association were due to a general effect of Bs on all the chromosome arms or to a particular effect on some arms. The results shown in Table 2 show that the only noticeable increase observed in the homologous association of long arms is due to an increase in the long arm of chromosome $3 \mathrm{R}$, the only long arm without a complete or almost complete association in plants without Bs. On the contrary, the increase in short arm association is produced by several chromosome arms. Significant increases occurred for the short arms of chromosome $3 R$ in all the plants with $B s, 1 R$ and $4 R$ in $14+2 B$ and $6 \mathrm{R}$ in $14+2 \mathrm{~B}$ and $14+$ iso-BS plants. The results in Table 2 also show that the non-significant differences observed in the association of the chromosome complement as a whole, or of the short arms, are the result of a compensatory effect between the increase and decrease in association in different chromosome arms. Thus, in plants with $14+2 \mathrm{~B}+$ iso- $\mathrm{BL}$ the increase in association in chromosome $3 \mathrm{R}$ is mainly compensated by the decrease in the short arms of chromosomes $1 \mathrm{R}$ and $7 \mathrm{R}$; likewise in plants with $14+$ iso-BL both significant increases and decreases are observed.

We have also analysed the homologous association between the two arms of the isochromosome B large (iso-BL) and the results are shown in Table 3. The nature of the isochromosome $\mathrm{B}$, long or short, was deduced from the similar length of its two arms and from the presence or absence in both arms of large blocks of telomeric constitutive heterochromatin. Figure 2 illustrates these aspects. 
a
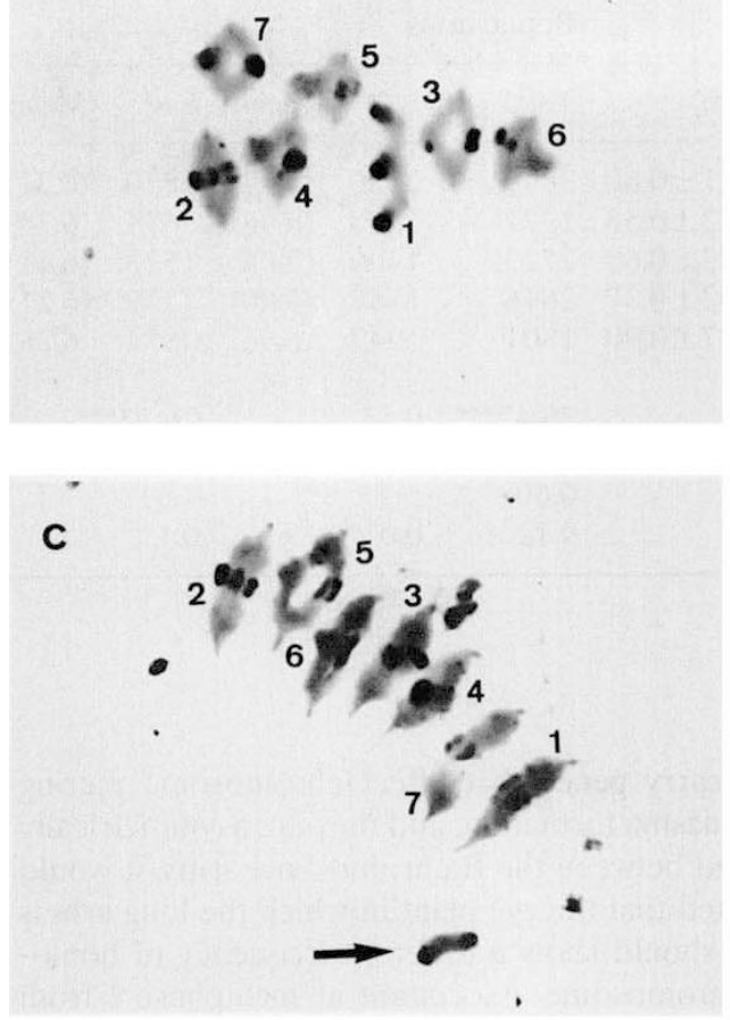

d

4

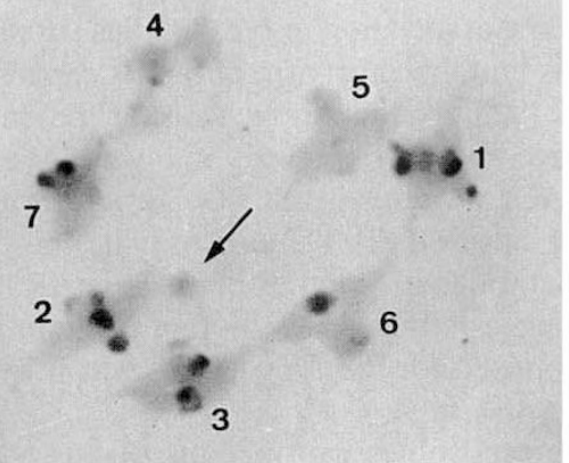

b

5
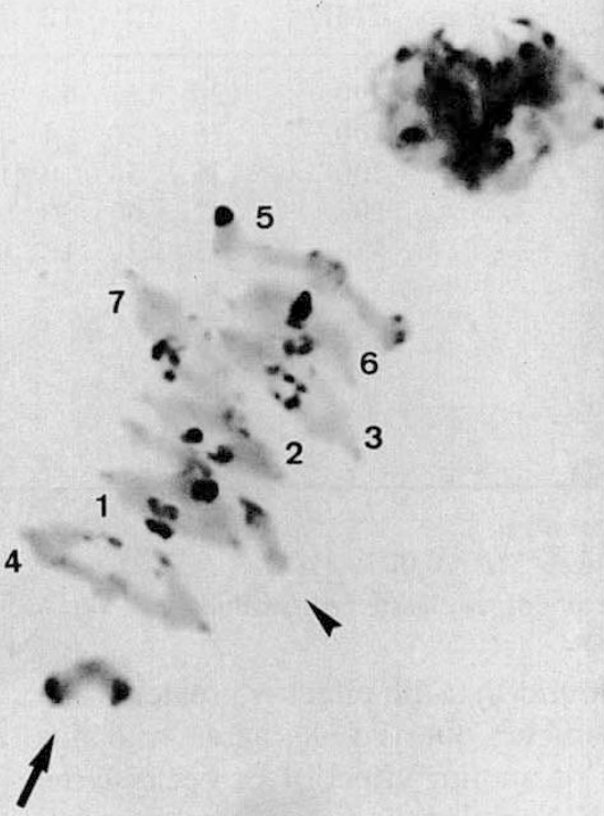

e

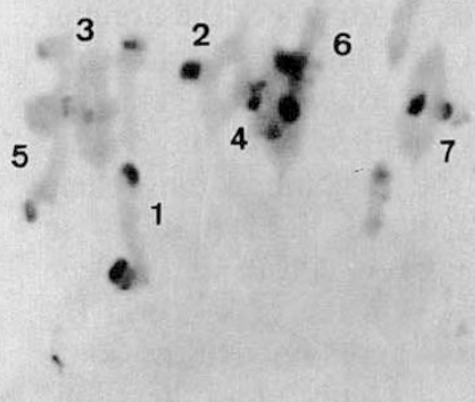

Fig. 1 Rye pollen mother cells at first metaphase from plants with different chromosome constitution. (a) 14 chromosomes, (b) 14 A chromosomes, 2 Bs a rod bivalent and an iso-BL as rod univalent, (c) $14+$ iso-BL, (d) $14+$ iso-BS, (e) $14+2 \mathrm{~B}$.

$\mathrm{B}$ isochromosomes are indicated by arrows and bivalents of B standard chromosomes by arrowheads; number indicates the respective pairs of A chromosomes.

\section{Discussion}

Data from previous works indicate that $B$ chromosomes can control both the level of chromosome pairing and the chiasma formation. It has been suggested that they can influence the meiosis of $A$ chromosomes by means of two opposite mechanisms: increasing or decreasing the chiasma formation.
It has been described in several species that the presence of Bs increases the chiasma frequency of As. In maize, Ayonoadu \& Rees (1968) showed that Bs increase the chiasma frequency; Rhoades \& Dempsey (1973) reported an effect that increased the recombination. Vosa \& Barlow (1972) detected a progressive average increase in chiasma number in relation to the increase in the number of Bs in Listera 
Table 1 Frequency distribution of A chromosome arms bound at metaphase I. Contingency $\chi^{2}$ of bound and unbound arms between plants with and without $\mathrm{B}$ chromosomes.

\begin{tabular}{|c|c|c|c|c|c|c|c|c|c|c|c|c|c|}
\hline \multirow{2}{*}{$\begin{array}{l}\text { Chromosome } \\
\text { constitution }\end{array}$} & \multirow{2}{*}{$\begin{array}{l}\text { Number } \\
\text { of plants }\end{array}$} & \multirow{2}{*}{$\begin{array}{l}\text { Number } \\
\text { CMP }\end{array}$} & \multicolumn{6}{|c|}{ Bound arms per cell } & \multicolumn{5}{|c|}{ Bound arms } \\
\hline & & & 14 & 13 & 12 & 11 & 10 & Mean & Total & $\mathrm{L}$ & (Mean) & $\mathrm{S}$ & (Mean) \\
\hline 14 & 3 & 290 & 120 & 117 & 47 & 5 & 1 & $13.21 \pm 0.80$ & 3849 & 2020 & $(6.97)$ & 1829 & $(6.31)$ \\
\hline $14+2 B$ & 1 & 100 & 76 & 20 & 4 & & & $13.72 \pm 0.53$ & 1372 & 698 & (6.98) & 674 & (6.74) \\
\hline $14+$ iso-BS & 2 & 200 & 136 & 54 & 9 & 1 & & $13.63 \pm 0.60$ & 2723 & 1400 & $(7.00)$ & 1323 & $(6.61)$ \\
\hline $14+2 B+$ iso-BL & 3 & 198 & 92 & 67 & 30 & 9 & & $13.22 \pm 0.87$ & 2616 & 1386 & $(7.00)$ & 1230 & (6.21) \\
\hline $14+$ iso- $\mathrm{BL}$ & 2 & 136 & 71 & 39 & 18 & 8 & & $13.27 \pm 0.90$ & 1801 & 947 & $(6.96)$ & 854 & (6.28) \\
\hline 14 vs. $14+2 B$ & & & & & & & & & $25.42^{* * *}$ & 0.52 & & $26.13^{*}$ & \\
\hline 14 vs. $14+$ iso-BS & & & & & & & & & $24.66^{* * *}$ & $-\dagger$ & & $21.59^{*}-x-1$ & \\
\hline 14 vs. $14+2 \mathrm{~B}+$ iso- $\mathrm{BL}$ & & & & & & & & & 0.60 & $-\dagger$ & & 1.61 & \\
\hline 14 vs. $14+$ iso-BL & & & & & & & & & 0.12 & 0.01 & & 0.11 & \\
\hline
\end{tabular}

$\mathrm{L}=$ long $\operatorname{arm} ; \mathrm{S}=$ short arm.

Chi-square significance, ${ }^{* * *} P<0.001$.

$\nmid$ Not scored because of empty classes (zero values).

ovata. They also found that the effect is greater when the Bs are odd numbers. Our results agree with this repeatedly alleged promoting effect of Bs because the average number of homologous association found is higher in the presence of Bs (Table 1).

On the other hand, it is worth emphasizing that an increase in the chiasma frequency within a bivalent is usually accompanied by a change in chiasma distribution (Darlington, 1965). Jones and Rees' (1967) work on rye supports this statement; they showed that the presence of Bs enhances the number of $\mathrm{A}$ bivalents with an increased number of chiasmata, simultaneously with a greater variability in A chiasma frequencies between pollen mother cells, the overall mean of chiasmata in all the $\mathrm{B}$ combinations being almost constant. In our case, if the variability in homologous association between cells and between bivalents within cells increases in the presence of $\mathrm{Bs}$, we expect that some bivalents and some chromosome arms have high frequencies of bonds, and others low. This seems to be the case for the short arms when plants with $2 \mathrm{~B}+$ iso- $\mathrm{BL}$ and iso- $\mathrm{BL}$ are compared with plants without any Bs (Table 2), while plants with $2 \mathrm{~B}$ and iso-BS show a general increase in most arms.

White \& Rees (1985), by using rye plants homozygous and heterozygous for chromosome interchanges and Parker et al. (1990) in Crepis capillaris, have shown that the effect of Bs on chiasma frequency can differ depending on the A chromosome. The analysis of individual chromosomes in the present study indicates that, at least for short arms, several A chromosomes but not all are implicated in the increase in chromosome association. This is particularly evident for $14+2 \mathrm{~B}$ and $14+$ iso-BS plants (Table 2).
If Bs carry genes that affect chromosome pairing and/or chiasma formation, and they are asymmetrically distributed between the B chromosome arms, it would be expected that the rye plant in which the long arm is included should show a different frequency of homologous chromosome association at metaphase I from plants in which the short arm is included. The results of our study agree with this assumption as the presence of the short arm induces an increase in homologous chromosome association in rye As, thus supporting the hypothesis that the short arm of the Bs carries promoting genes. Results that show that rye Bs carry these genes have been observed in wheat-rye hybrids (Romero \& Lacadena, 1980; Cuadrado et al., 1988). Jenkins \& Scanlon (1987), on studying the formation of the synaptonemal complex in interspecific hybrids of Lolium, suggested that the action of Bs is similar to the action proposed for the $P h$ genes of wheat by Holm \& Whang (1988).

B-isochromosomes can pair in meiosis with standard Bs or with themselves to form a ring univalent (Kishikawa, 1977). It seems logical to expect that plants with standard Bs and single B-isochomosomes should show multivalent associations with a high frequency, whereas plants with only single B-isochromosomes should show a high frequency of iso-BL ring univalents. However, the actual results obtained for our plants are the opposite: while in plants with two standard $\mathrm{Bs}$ and an iso-BL, the isochromosome appears as a ring univalent or associated with the $\mathrm{B}$ standard to form a multivalent (Fig. 2), in plants without the B standard the isochromosome appears as a rod univalent in $98.5 \%$ of the cells (Table 3 ). These results indicate that the presence of standard Bs enhances the 

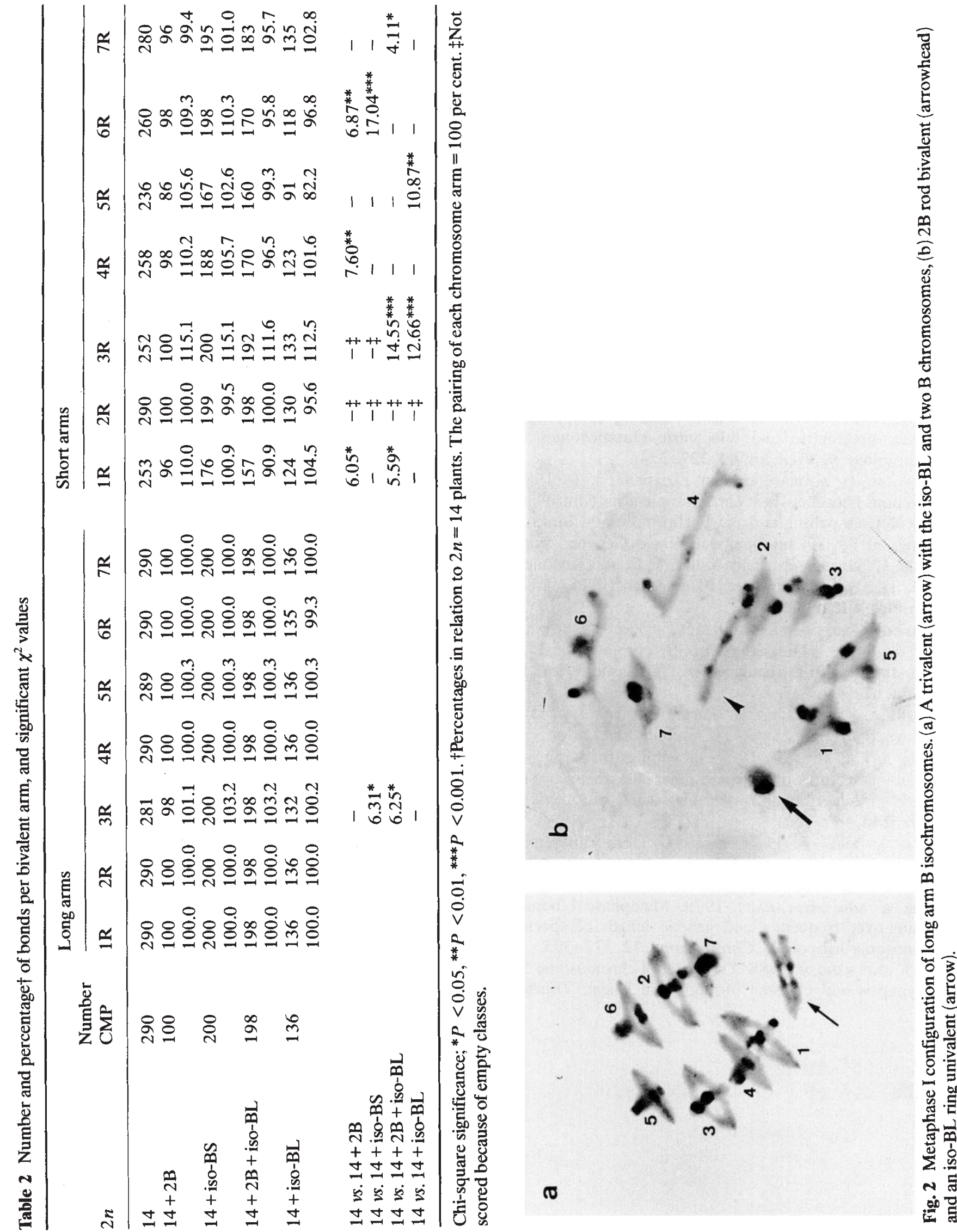
Table 3 Association between the two arms of the iso B large at metaphase I

\begin{tabular}{llll}
\hline $\begin{array}{l}\text { Type of } \\
\text { B chromosomes }\end{array}$ & $\begin{array}{l}\text { Ring B } \\
\text { univalent }\end{array}$ & $\begin{array}{l}\text { Rod B univalent } \\
\text { or bound in multivalents }\end{array}$ & $\begin{array}{l}\text { Percentage of self } \\
\text { association }\end{array}$ \\
\hline 2B + iso-BL & 56 & 142 & 28.28 \\
iso-BL & 2 & 134 & 1.47 \\
\hline
\end{tabular}

association between the two identical arms of the B-isochromosome. They also support the previously observed presence of promoting genes in the Bs short arm.

\section{References}

AYONOADU, V. AND REES, H. 1968. The influence of B chromosomes on chiasma frequencies in black Mexican sweet corn. Genetica, 39, 75-81.

benavente, E. AND orellana, J. 1989. Pairing competition between metacentric and telocentric chromosomes in autotetraploid rye. Heredity, 62, 327-334.

CUADRADO, M. C., ROMERO, C. AND LACADENA, J. R. 1988 Interactions between wheat chromosome arms controlling heomeologous pairing and rye B chromosomes. In: Proceedings of the 7 th International Wheat Genetics Symposium, Cambridge, England. Miller, T. E. and Koebner, R. M. D. (eds). Institute of Plant Sciences Research, Cambridge, UK, pp. 237-241.

CRUZ-PARDIlla, M., VENCES, F. J., GARCIA, P. AND PEREZ DE LA VEGA, M. 1989. The effect of B chromosomes on outcrossing rate in a population of rye, Secale cereale $\mathrm{L}$. Heredity, 62, 319-325.

DARlington, C. D. 1965. Cytology. Part II: Recent Advances in Cytology, 1937-1964. J. and A. Churchill Ltd. London.

DOVER, G. A. AND RILEY, R. 1972. Prevention of pairing of homoeologous meiotic chromosomes of wheat by an activity of supernumerary chromosomes of Aegilops. Nature, 240, 159-161.

FOMINAYA, A. AND OREllaNA, J. 1988. Does differential C-heterochromatin content affect chromosome pairing in octoploid triticale. Heredity, 61, 167-173

GiRAldeZ, R. AND orellana, J. 1979. Metaphase I bonds, crossing-over frequency and genetic length of specific chromosome arms of rye. Chromosoma, 72, 377-385.

HOLM, P. B. AND WANG, w. 1988. The effect of chromosome 5B on synapsis and chiasma formation in wheat, Triticum aestivum cv. Chinese Spring. Carlsberg Res. Comm., 53, $191-208$

JENKINS, G. AND SCANLON, M. J. 1987. Chromosome pairing in a Lolium temulentum $\times$ Lolium perenne diploid hybrid with a low chiasma frequency. Theor. Appl. Genet., 73, 516-522.

JONES, R. N. AND REES, H. 1967. Genotypic control of chromosome behaviour in rye. XI. The influence of $B$ chromosomes on meiosis. Heredity, 22, 333-347.

JONES, R. N. AND REES, H. 1982. B Chromosomes. Academic Press, London.

KISHIKAWA, H. 1977. Effect of the small iso B and deficient B chromosomes on the pairing of standard B chromosomes in rye. Cytologia, 42, 315-322.

ORELLANA, J. AND GIRALDEZ, R. 1981. Metaphase I bound arms and crossing over frequency in rye $\mathrm{I}$. Open pollinated varieties. Chromosoma, 84, 439-449.

PARKER, J. S., JONES, G. H., EDGAR, L. A. AND WHITEHOUSE, C. 1990. The population cytogenetics of Crepis capillaris. III. B-chromosome effects on meiosis. Heredity, 64, 377-385. RHOADES, M. M. AND DEMPSEY, E. 1973. Chromatin elimination induced by the $\mathrm{B}$ chromosome of maize. I. Mechanism of loss and the pattern of endosperm variegation. J. Hered., 64, 13-18.

ROMERO, C. AND LACADENA, J. R. 1980. Interaction between rye B chromosomes and wheat genetic system controlling homeologous pairing. Chromosoma, 80, 33-48.

TSUMOTO, L. AND SASAK1, M. 1972. Effect of B chromosomes on chiasma frequency of A chromosomes in rye. Wheat Inf. Serv., 33-34, 28-30.

vosA, C. G. AND BARLow, P. W. 1972. Meiosis and B chromosomes in Listera ovata (Orchidaceae). Caryologia, 25, $1-8$.

WHITE, J. AND REES, H. 1985. Chromosome specific control of chiasma frequency in rye. Heredity, 55, 421-426.

ZARCHI, Y., SIMCHEN, G., HILLEL, J. AND SCHAPP, T. 1972. Chiasmata and the breeding system in wild populations of diploid wheats. Chromosoma, 38, 77-94. 